\title{
Revenue Adequacy Constrained Optimal Transmission Switching
}

\author{
Kory W. Hedman \\ Arizona State University \\ kory.hedman@asu.edu
}

\author{
Shmuel S. Oren \\ University of California, Berkeley \\ oren@ieor.berkeley.edu
}

\author{
Richard P. O’Neill \\ Federal Energy Regulatory Commission \\ richard.oneill@ferc.gov
}

\begin{abstract}
Financial Transmission Rights (FTRs) are used to hedge congestion risk and they are financed by congestion rents. The ISO may not collect enough congestion rents to cover its obligation to the FTR holders; this is known as revenue inadequacy. Revenue inadequacy may occur even though ISOs run a Simultaneous Feasibility Test (SFT), which ensures revenue adequacy. However, the SFT relies on the assumption that the grid topology is not modified. Recent research suggests that we should co-optimize generation with the network topology. There is the concern that optimizing the topology will cause revenue inadequacy. In this paper, we examine how transmission switching affects revenue adequacy of FTRs. We discuss how the optimal transmission switching problem can be modified in order to maximize the market surplus subject to maintaining revenue adequacy, if that is the desired motivation of the ISO. We also discuss the policy implications of adopting such a method.
\end{abstract}

\begin{tabular}{ll}
\multicolumn{1}{c}{ Nomenclature } \\
\multicolumn{1}{c}{ Indices and } & Sets \\
$g(n):$ & Generator. \\
$k:$ & Set of generators at bus $n$. \\
$k(n,),. k(., n):$ & Transmission element (line or transformer). \\
& Set of transmission elements with bus $n$ as the \\
& to bus and the set with bus $n$ as the from bus. \\
$K 0^{i}:$ & Set of opened lines for network topology \\
& solution $i$ that has an optimal solution in the \\
& Benders' sub-problem but yet is revenue \\
$K 1^{i}:$ & inadequate. \\
& Set of closed lines for network topology \\
& solution $i$ that has an optimal solution in the \\
& Benders' sub-problem but yet is revenue \\
$m, n:$ & inadequate. \\
Parameters & Nodes. \\
$B_{k}:$ & Susceptance of transmission element $k$. \\
$c_{g}:$ & Production cost for generator $g$. \\
$d_{n}:$ & Real power load at node $n$. \\
$F T R^{n m}{ }_{f}:$ & Financial transmission right contract $f$ \\
$M_{k}:$ & between sink bus $n$ and source bus $m$. \\
& Big M value for transmission element $k$.
\end{tabular}

\begin{tabular}{|c|c|}
\hline $\begin{array}{l}P_{a}^{\max }: \\
P_{k}^{\max }, P_{k}^{\min }:\end{array}$ & $\begin{array}{l}\text { Max capacity of generator } g \text {. } \\
\text { Max and min rating of transmission element } k \text {; } \\
\text { note that, generally, } P_{k}^{\max }=-P_{k}^{\min } \text {. }\end{array}$ \\
\hline$F_{k}^{+}, F_{k}^{-}:$ & $\begin{array}{l}\text { Dual variables on line } k \text { 's capacity constraints, } \\
\text { upper bound and lower bound respectively } \\
\text { (flowgate marginal prices). }\end{array}$ \\
\hline$L M P_{n}:$ & $\begin{array}{l}\text { Dual variable on bus n's power balance } \\
\text { constraint (Locational marginal price). }\end{array}$ \\
\hline$P_{g}:$ & Real power supply from generator $g$ at node $n$. \\
\hline$P_{k}$ : & $\begin{array}{l}\text { Real power flow from node } m \text { to } n \text { for } \\
\text { transmission element } k \text {. }\end{array}$ \\
\hline$z_{k}:$ & $\begin{array}{l}\text { Binary switching variable for transmission } \\
\text { element } k(0 \text { open/not in service, } 1 \text { closed/in } \\
\text { service })\end{array}$ \\
\hline$\alpha_{g}:$ & Dual variable on generator $g$ 's upper bound. \\
\hline$\theta_{n}:$ & Bus voltage angle at node $n$ \\
\hline$\mu_{k}^{+}, \mu_{k}^{-}$: & $\begin{array}{l}\text { Dual variables on the relationship between } \phi_{k} \text {, } \\
\theta_{n} \text {, and } \theta_{m} \text {. }\end{array}$ \\
\hline$\phi_{k}:$ & $\begin{array}{l}\text { Bus voltage angle difference across } \\
\text { transmission element } k \text {. }\end{array}$ \\
\hline$\omega$ & $\begin{array}{l}\text { Master problem objective in the Benders' } \\
\text { decomposition technique. }\end{array}$ \\
\hline
\end{tabular}

\section{Introduction}

Point-to-point Financial Transmission Rights (FTRs) are common in many restructured electric energy markets and they allow market participants to hedge forward contracts or speculate on price differences. FTRs are instrumental in the electric energy markets since they enable allocation of property rights to the electric power grid and management of congestion risk by energy market participants without inhibiting efficient operation of the grid by the system operator. An FTR entitles the owner to the difference in the Locational Marginal Prices (LMPs) between the defined source and sink locations, i.e., the sink price minus the source price, times the quantity of the FTR. In electric energy markets that use a nodal pricing system, congestion charges are equal to the LMP difference between the withdrawal (sink) point and the injection (source) point times the flow. An FTR, therefore, allows a market participant to create a perfect hedge against the congestion charges for 
sending one MW of power between these two assigned nodes. Such a financial instrument can be contracted to be a two sided obligation, meaning that the holder of the FTR is obligated to cover the financial position no matter if it is positive (FTR holder is paid) or negative (FTR holder must pay), or the contract can be structured as an option based financial instrument. FTRs are typically obligatory contracts and, thus, FTRs will be assumed to be obligations throughout this paper.

In most electric energy markets that operate an FTR market, the Independent System Operator (ISO) auctions off the FTRs to market participants with the objective to maximize the auction revenues. However, the allocation of all FTRs for the network must pass what is called a Simultaneous Feasibility Test (SFT). The SFT guarantees that if all FTR positions are physically exercised simultaneously that the electrical network can handle all physical flows throughout the network, i.e., no network constraint violations, based on the FTRs' defined injection point, withdrawal point, and the defined transfer quantity.

The FTRs holders are compensated by the ISO, which covers this financial obligation with the congestion rent that is collected from the market. Revenue adequacy occurs when the ISO has enough congestion rent to fully cover its obligation to FTR holders. Revenue inadequacy occurs when the ISO does not have adequate congestion rents to fully compensate the FTR holders. It was shown in [19] that under certain theoretical conditions, the congestion rent collected by the ISO will be adequate to cover all obligations for any FTR allocation that satisfies the SFT. The proof that there will be revenue adequacy relies on the "separating hyperplane principle" of convex optimization and the assumption that the SFT feasible set (nomogram) is convex.

The feasible set of solutions for the DC load flow model, which is an approximation of the AC load flow model, is represented by a convex polyhedron. Thus, the DC load flow model can satisfy this assumption and revenue adequacy can be guaranteed for the DC load flow model. Conversely, the feasible set of solutions for the actual flows in the electric grid, which are based on the AC load flow model, is not convex; thus, the SFT cannot guarantee revenue adequacy with an AC load flow model.

Revenue inadequacy can also occur when lines' thermal capacities are de-rated for various operational reasons. Furthermore, revenue adequacy is maintained for the static DC network [27] but it is not guaranteed if the network topology changes [1]. Revenue inadequacy can, therefore, occur when there is a transmission outage within the system that was not modeled in the SFT.
As a result, the FTR markets are often revenue inadequate. However, there can also be situations where there is a revenue surplus, meaning that there is more congestion rent than the total of all FTR settlements. Since the ISOs settle the FTR positions over a longer time horizon, e.g., one month, this helps reduce the severity of revenue inadequacy. Even still, FTR markets are commonly revenue inadequate and the various ad hoc protocols used today to deal with revenue inadequacy all create at least one, if not more, loser. One common approach used by many ISOs is to uniformly de-rate the payments to the FTR holders when there is revenue inadequacy. Such a protocol obviously decreases the FTRs' value; it also undermines the purpose of such a financial instrument, which is used to hedge price risk.

There is currently a national push to create a smarter, more flexible grid. In particular, research has demonstrated the potential benefit of creating a more flexible transmission grid through the use of Flexible AC Transmission System (FACTS) devices, developing dynamic thermal line ratings, incorporation of transmission switching into economic dispatch problems, etc. As new technologies and operating procedures are incorporated into grid operations, current market mechanisms and protocols may be undermined by such advances. This paper focuses on the smart grid concept of co-optimizing the network topology with the generation, how this concept may affect revenue adequacy within the FTR market, and, finally, this paper presents the concept of revenue adequacy constrained network topology optimization, a method that co-optimizes the network topology with generation like past research but modifies the optimization problem to ensure that there is revenue adequacy within the FTR markets. This research is based on the Direct Current Optimal Power Flow (DCOPF) model and our discussion on revenue adequacy refers only to changing the network topology and does not include other possible causes of revenue inadequacy mentioned above.

First, Section 2 presents an overview of the optimal transmission switching concept and how it affects the feasible set of dispatch solutions as compared to when the topology is treated as a fixed, static asset. Section 2 also presents theoretical examples on how optimal transmission switching affects the feasible region of the SFT test. Section 3 then presents the optimal transmission switching DCOPF formulation. Section 4 provides a discussion on how this mixed integer program can be modified to ensure that there is revenue adequacy in this side financial market, the FTR market. This section also presents the revenue adequacy constrained optimal transmission switching formulation. Section 5 presents 
a test case and the corresponding results. The following section, Section 6, discusses the policy implication of the general problem of dealing with revenue inadequacy as well as a discussion on the presented technique as a mechanism to deal with revenue inadequacy caused by network topology optimization. Finally, we discuss future research in Section 7 and Section 8 concludes this paper.

\section{Transmission switching, feasible regions, and revenue adequacy}

\subsection{Overview of transmission switching}

Traditionally, transmission networks for bulk power flow have been modeled as static, except during times of forced outages or maintenance. This traditional view does not describe them as assets that operators have the ability to control. However, system operators can and do change the network topology to improve system performance. The flow of electric energy across the grid is governed by unique physical laws and by taking a line temporarily out of service this can change flows throughout the network. As a result, by harnessing the control over a transmission line's status, one can gain additional operational flexibility in the short run, which can be used to improve a variety of different grid operations.

The concept of transmission switching as a corrective mechanism has existed for many years. Transmission switching has been proposed as a corrective mechanism for problems such as over or under voltage violations, line overloading, and system security, see [2], [4], [11], [12], [21], [31]-[34]. Transmission switching can also be used to help reduce system losses, see [3] and [10], and it can be used as a congestion management tool as well, [13]. One potential setback of transmission switching is its impact on transient stability; however, [5] has shown that switching actions can actually help improve the transient stability of the network. Furthermore, transmission switching can be conducted in conjunction with transient stability constraints in order to ensure that the switching actions do not cause a transient stability problem, [6].

Moreover, various ad-hoc transmission switching procedures exist today, thereby confirming that it is indeed possible to harness the flexibility of the transmission grid while maintaining reliability and not causing stability concerns. During certain operational situations, it is possible to temporarily take a transmission element out of service to increase transfer capacity or improve voltage profiles [28]. For example, it is an accepted practice to improve voltage profiles by opening light-loaded transmission lines at night [22]. The Northeast Power Coordinating Council includes "switch out internal transmission lines" in the list of possible actions to avoid abnormal voltage conditions, see [20] and [24].

Numerous Special Protection Schemes (SPSs) address specific instances of switching during steadystate conditions as well as during emergency conditions. PJM has implemented SPSs that involve both pre-contingency and post-contingency switching actions, [29]. Certain SPSs allow the operator to disconnect a line during normal operations but return it to service if a contingency occurs. Similarly, there are SPSs that involve the opening of a line after a contingency occurs in order to avoid specific network constraint violations.

Even though past research has identified the benefits from harnessing the flexibility of transmission assets, traditional security constrained economic dispatch of electricity resources still treats the transmission network as a fixed and static topology while optimizing deployment of generation assets. However, it is well known that the redundancy built into the grid in order to handle the multitude of contingencies over a long planning horizon can, in the short run, create congestion and necessitate costly out of merit dispatch. While it is quite common for operators to occasionally open lines, such practices are employed on an ad hoc basis and are not driven by cost considerations. Furthermore, such options are not included, formally, in the classical formulation of dispatch optimization problems common in system and market operation procedures employed by vertically integrated utilities and Independent System Operators (ISOs).

Accordingly, recent research has proposed that day-ahead economic dispatch models should account for the flexibility of transmission assets. The concept of a dispatchable transmission network was investigated by [25]. Optimal transmission switching has been shown to produce substantial savings for various test cases, [8], [14]-[18], [26]. Even though this concept takes transmission lines temporarily out of service for economic gains, optimal transmission switching is not incompatible with reliable network operations. Indeed, [14] and [16] demonstrated that transmission switching can be beneficial even while ensuring an N-1 reliable network.

While optimal transmission switching has the objective to maximize the market surplus, it creates winners and losers among market participants and it can cause revenue inadequacy in the FTR markets. The following two subsections discuss how optimal transmission switching affects the feasibility set of 
dispatch solutions and how it affects the set of feasible FTR allocations, which is determined by the SFT.

\subsection{Transmission switching and the feasible set}

The feasible set of dispatch solutions depends on the topology, the impedances of the lines, as well as other network flow constraints. By opening a transmission line, this changes the feasible set and, thus, optimal transmission switching provides a feasible set that is a superset of the feasible region when transmission lines are treated as static assets. Below is a simple 3-bus example shown by Figure 1. The feasible sets in Figure 2 are defined by transmission constraints. For the original topology, there are three equations that represent the network constraints, (1)-(3); only the constraints that define the feasible sets are shown in Figure 2. With all lines closed, the feasible set is defined by the set of vertices $\{0,1,2,3\}$ in Figure 2. If line A-B is opened, the feasible set is $\{0,4,5,6\}$.

$$
\begin{aligned}
& -180 \leq G_{A}-G_{B} \leq 180 \\
& -300 \leq G_{A}+2 G_{B} \leq 300 \\
& -240 \leq 2 G_{A}+G_{B} \leq 240
\end{aligned}
$$

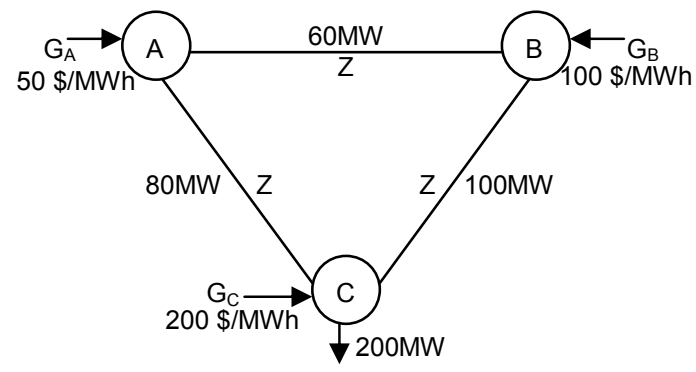

Figure 1. 3-Bus Example

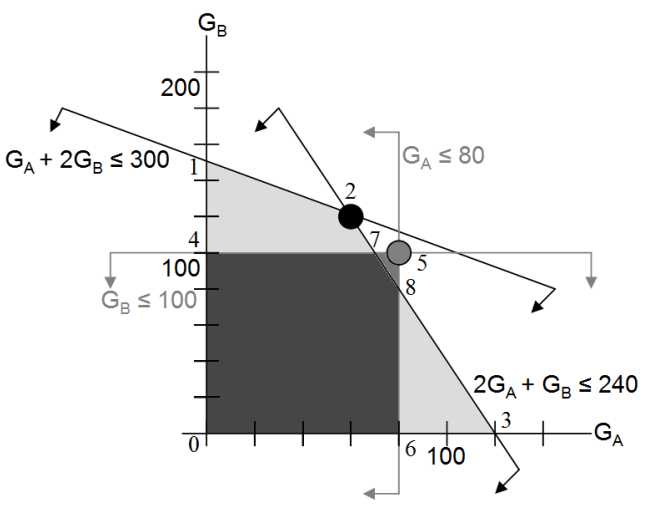

Figure 2. Gen A and Gen B Feasible Sets for 3Bus Example

The feasible set for generators $\mathrm{A}$ and $\mathrm{B}$ with optimal transmission switching is $\{0,1,2,7,5,8,3\}$, which is not convex. Since the original topology can always be chosen, there is the guarantee that the objective will never be worse than before. The optimal solution for generators $\mathrm{A}$ and $\mathrm{B}$ with the original topology is at the black dot, vertex two; the dispatch levels are: 60, 120, and 20 for generators $\mathrm{A}, \mathrm{B}$, and $\mathrm{C}$ respectively with a cost of $\$ 19,000$. With optimal transmission switching, the optimal solution is at the grey dot, vertex five; the dispatch levels are: 80, 100, and 20 for generators $\mathrm{A}, \mathrm{B}$, and $\mathrm{C}$ respectively and the optimal cost is $\$ 18,000$.

\subsection{Optimal transmission switching and the simultaneous feasibility test}

Continuing with this 3-bus example, if we define two FTR positions, from source A to sink C (FTR $\left.\mathrm{FC}_{\mathrm{AC}}\right)$ and from source $B$ to sink $C\left(F R_{B C}\right)$, then (1a)-(3a) define the feasible set for FTR positions that would pass the SFT with all lines in service. If line A to B is open, then the feasible set is defined by (4) and (5). Note that these are the exact same equations as the ones represented in Figure 2, except that $G_{A}$ is replaced with FTR $_{\mathrm{AC}}$ and $\mathrm{G}_{\mathrm{B}}$ is replaced with FTR $\mathrm{BC}_{\mathrm{BC}}$. Thus, by examining Figure 2, it is clear that if the set of FTRs for the system is in the sets defined by $\{4,1,2,7\}$ or $\{6,8,3\}$, then revenue adequacy would not be guaranteed when line A-B is open as those FTR allocations would not pass the SFT for the reconfigured topology. Furthermore, if line A-C is opened, (6) and (7) define the feasible set and if line B-C is opened, (8) and (9) define the feasible set.

$$
\begin{aligned}
& -180 \leq F T R_{A C}-F T R_{B C} \leq 180 \\
& -300 \leq F T R_{A C}+2 F T R_{B C} \leq 300 \\
& -240 \leq 2 F T R_{A C}+F T R_{B C} \leq 240 \\
& -80 \leq F T R_{A C} \leq 80 \\
& -100 \leq F T R_{B C} \leq 100 \\
& -60 \leq F T R_{A C} \leq 60 \\
& -100 \leq F T R_{A C}+F T R_{B C} \leq 100 \\
& -60 \leq F T R_{B C} \leq 60 \\
& -80 \leq F T R_{A C}+F T R_{B C} \leq 80
\end{aligned}
$$

\section{Optimal transmission switching DCOPF problem}

Equations (10)-(14) define the Direct Current Optimal Power Flow (DCOPF) problem. This DCOPF formulation is slightly different than other DCOPF formulations due to the way that we modify the DCOPF to incorporate transmission switching. We 
have also introduced a new variable, $\phi_{k}$. For the purpose of this paper, we assume that generators' cost functions are linear, i.e. constant marginal cost ${ }^{1}$, and, hence, the DCOPF problem is a linear program.

$$
\begin{aligned}
& \text { Minimize: } \sum_{g} c_{g} P_{g} \\
& \text { s.t.: } P_{k}^{\min } \leq B_{k} \phi_{k} \leq P_{k}^{\max }, \forall k \\
& -\phi_{k}+\left(\theta_{n}-\theta_{m}\right)=0, \forall k \\
& \sum_{\forall k(n, .)} B_{k} \phi_{k}-\sum_{\forall k(., n)} B_{k} \phi_{k}+\sum_{\forall g(n)} P_{g}=d_{n}, \forall n \\
& 0 \leq P_{g} \leq P_{g}^{\max }, \forall g
\end{aligned}
$$

We first introduce a new binary variable, $z_{k}$, which equals one if the line is in service (breakers are closed) and zero if it is out of service (breakers are open). The first modification that is made to the traditional DCOPF is that the line's upper and lower bounds are multiplied by this binary variable to force the line's flow to equal zero if it is not in service; this is represented by (11'). We also modify (12) to create (12a) and (12b). With this modification, when $z_{k}$ equals one, (12a) and (12b) will enforce (12). When $z_{k}$ equals zero, the use of the large multiplier $M_{k}$ makes it such that $\theta_{n}-\theta_{m}$ can be different from $\phi_{k}$. The only restriction on $\theta_{n}-\theta_{m}$ when the line is open comes from the value of $M_{k}$. These lines will only be taken out of service temporarily and, thus, they will be placed back into service. Larger angle separations can cause additional stress on the breaker equipment during the reclosing procedure and, thus, the value of $M_{k}$ is determined based on this operational restriction. Finally, (15) reflects the fact that $z_{k}$ is a binary variable.

$$
\begin{aligned}
& P_{k}^{\min } z_{k} \leq B_{k} \phi_{k} \leq P_{k}^{\max } z_{k}, \forall k \\
& -\phi_{k}+\left(\theta_{n}-\theta_{m}\right)+\left(1-z_{k}\right) M_{k} \geq 0, \forall k \\
& -\phi_{k}+\left(\theta_{n}-\theta_{m}\right)-\left(1-z_{k}\right) M_{k} \leq 0, \forall k \\
& z_{k} \in\{0,1\}, \forall k
\end{aligned}
$$

\section{Revenue adequacy constrained optimal transmission switching}

\subsection{Revenue adequacy test}

\section{In Section 2.3 it was shown how optimal}

\footnotetext{
1 In reality, generator cost functions are generally modeled as quadratic in output (aside from startup and no load costs); however, in practice, such cost functions are approximated by piecewise linear functions represented as block offers at different marginal prices. The DCOPF formulation with piecewise linear cost functions is also a linear programming problem.
}

transmission switching affects the SFT and, thus, may cause revenue inadequacy. One potential solution is to alter the optimal transmission switching problem to choose only a topology that both increases the market surplus, as compared to the case when transmission assets are treated as fixed assets, and ensures that the FTR market is revenue adequate. This option, however, is a controversial option as the market participants would be "leaving money on the table" in the sense that the grid operator could then be passing up a solution that provides more market surplus in order to ensure revenue adequacy in this side financial market, the FTR market. Nevertheless, this section presents this altered formulation, the revenue adequacy constrained optimal transmission switching problem. The following section discusses the policy implications related to dealing with revenue inadequacy and this concept.

A solution is revenue adequate if the congestion rent is at least as large as the total FTR settlements. Equation (16) can be used to test if a network is revenue adequate; the left hand side of (16) represents the load payment minus the generation revenue, which is equal to the congestion rent (note that this is a lossless model). The right hand side of (16) represents the FTR payment where $F T R^{n m}{ }_{f}$ reflects FTR contract $f$ between the source bus $m$ and the sink bus $n$.

$\sum_{n} L M P_{n}\left(d_{n}-\sum_{g(n)} P_{g}\right) \geq \sum_{f}\left(L M P_{n}-L M P_{m}\right) F T R_{f}^{n m}(16)$

The congestion rent is also defined as the line's flow times the dual variable on its capacity constraint, which is also known as the flowgate marginal price. With this definition and by using complementary slackness, (16) can be rewritten as (16a) below. The left hand side can also be identified as the congestion rent as it appears in the dual's objective. The dual's objective has the identity: load payment minus generation rent minus congestion rent. Each of these terms are easy to identify in the dual's objective with the congestion rent defined as the left hand side of (16a). We also introduce $\delta$, which is a parameter and can be used to specify that the solution must be revenue adequate, i.e., $\delta=1.0$, or $\delta$ can be set to be less than one thereby allowing for the possibility of revenue inadequacy but limiting the severity of revenue inadequacy. From now on, we will assume that $\delta=1.0$.

$\sum_{k} P_{k}^{\max }\left(F_{k}^{+}+F_{k}^{-}\right) \geq \delta \sum_{f}\left(L M P_{n}-L M P_{m}\right) F T R_{f}^{n m}$

The optimal transmission switching problem is a Mixed Integer Programming (MIP) problem; the dual of a MIP is not well defined. In order to know if a solution is revenue adequate, it is necessary to know the dual variables, i.e., the LMPs, that come from the 
OPF problem. As a result, it is not possible to simply add constraints to the original optimal transmission switching problem to ensure that revenue adequacy is maintained. Thus, to implement this check for revenue inadequacy, we instead examine solution techniques for MIP problems and determine how to modify these solution techniques to incorporate the requirement that the chosen topology is revenue adequate.

MIPs are commonly solved by branch and bound. Such an algorithm could be modified to implement a test to check for revenue adequacy. Instead of modifying branch and bound, we use a decomposition technique where it is possible to test whether topology solutions are revenue adequate. Benders' decomposition is a well known decomposition technique for MIPs; a description of Benders' decomposition can be found in [23]. One particular reason why it may be a good choice to use Benders' decomposition is because it is a row generation technique and, thus, it can be used to exploit the special structure that exists in economic dispatch problems that include contingency modeling in order to ensure that the system is N-1 reliable. Furthermore, additional techniques can be used to speed up the solution time, [7], [9], and [30].

Benders' decomposition separates the MIP into a master problem, an integer program, and a subproblem, a linear program. The master program will generate a solution for the binary variables and then pass that solution on to the sub-problem. With all integer variables fixed based on the previous master problem iteration, the MIP is then an LP and the dual of this LP is then represented by the sub-problem.

Figure 3 provides the outline of the Benders' decomposition technique along with how it is modified to ensure that the topology solution is revenue adequate. If the dual sub-problem is unbounded, the typical Benders' ray cut is applied and there is no need to check for revenue adequacy. If the dual sub-problem has an optimal solution, then instead of applying the typical Benders' optimality cut, we first check to see if there is revenue inadequacy by checking if (16a) holds. If (16a) holds, this particular network topology solution is revenue adequate and the Benders' decomposition technique will continue as planned by applying the traditional Benders' optimality cut. If the solution is not revenue adequate, a cut is generated that will be applied to the master problem that makes that specific integer solution, i.e., that specific network topology, infeasible. The cut, (17), is based on the sets listed by (18) that are determined from the current master problem iteration.

This process is repeated until the upper and lower bounds converge, thereby proving optimality of the original MIP problem. Modifying the Benders' decomposition technique in this way does not break the guarantee of optimality because whenever (17) is applied, that topology solution can never be chosen again by the master problem. In addition, (17) is a cut that does not affect any other, feasible or infeasible, network topology solution. In situations where the topology with all lines closed cannot be chosen, it is possible that no solution exists; only then is it possible for the algorithm to specify that the original MIP program is infeasible.

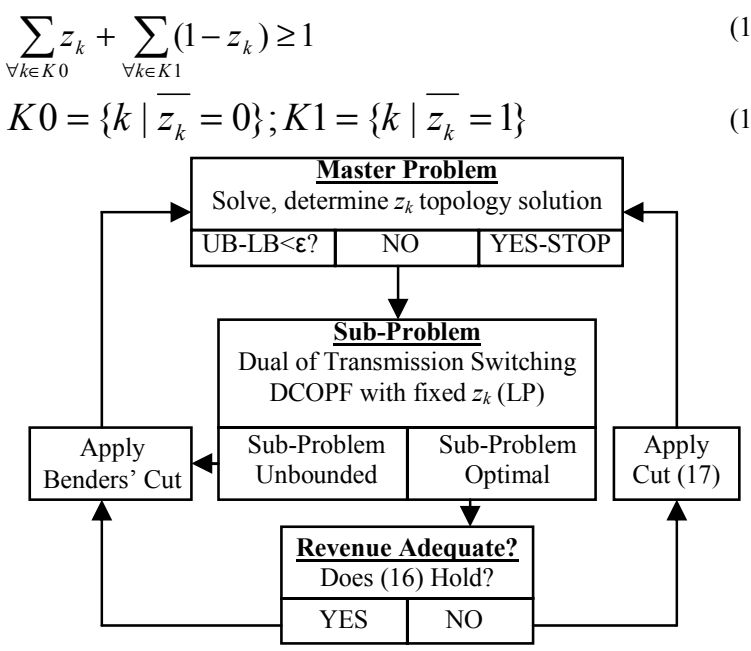

\section{Figure 3. Benders' decomposition technique to ensure revenue adequacy with optimal transmission switching}

\subsection{Benders' decomposition}

The optimal transmission switching DCOPF formulation is rewritten below with the integer variables fixed to their optimal values and its corresponding dual is presented as well, which is represented by the sub-problem in the Benders' decomposition technique outlined below, see (26)-(30). The master problem for the Benders' decomposition technique is presented by (20)-(25). In the master problem, (21) is repeated for all $i \in I$, which references the combinatorial cut that is applied to the master problem each time there is a feasible topology solution that is revenue inadequate; this cut that is applied to the master problem depends on the sets defined in (25). For each iteration where the network topology produces an optimal solution in the subproblem and this particular network topology is revenue inadequate, sets $K 0^{i}$ and $K 1^{i}$ are generated and they represent the transmission elements that are opened and closed respectively. Each time the subproblem has an optimal solution and the network topology is revenue adequate, or for each $p \in P$ ( $p$ represents extreme point solutions from the sub- 
problem), then the traditional Benders' optimality cut is applied as shown by (22). Likewise, whenever the sub-problem is unbounded, or for each $r \in R$ ( $r$ represents unbounded rays from the sub-problem), the traditional Benders' unbounded ray cut is applied to the master problem, as noted by (23). Note that there always exists a feasible solution to the sub-problem, as there is always a solution corresponding to allowing all dual variables to take on a value of zero since $c_{g}$ is nonnegative. A description of each step in the Benders' decomposition technique is listed after the subproblem.

$$
\begin{aligned}
& \text { Minimize: } \sum_{g} c_{g} P_{g} \\
& \text { s.t.: }-B_{k} \phi_{k} \geq-P_{k}^{\max } \overline{z_{k}}, \forall k \\
& B_{k} \phi_{k} \geq-P_{k}^{\max } \overline{z_{k}}, \forall k \\
& -\phi_{k}+\left(\theta_{n}-\theta_{m}\right) \geq-\left(1-\overline{z_{k}}\right) M_{k}, \forall k \\
& \phi_{k}-\left(\theta_{n}-\theta_{m}\right) \geq-\left(1-\overline{z_{k}}\right) M_{k}, \forall k \\
& \sum_{\forall k(n, .)} B_{k} \phi_{k}-\sum_{\forall k(., n)} B_{k} \phi_{k}+\sum_{\forall g(n)} P_{g}=d_{n}, \forall n \\
& -P_{g} \geq-P_{g}^{\max }, \forall g \\
& P_{g} \geq 0 ; \phi_{k}, \theta_{n} \text { free }
\end{aligned}
$$

$z_{k} \in\{0,1\} ; \omega$ free

where

$$
K 0^{i}=\left\{k \mid{\overline{z_{k}}}^{i}=0\right\} ; K 1^{i}=\left\{k \mid{\overline{z_{k}}}^{i}=1\right\}, \forall i \in I
$$

Sub-problem: Maximize: $\sum_{n} d_{n} L M P_{n}-\sum_{k} P_{k}^{\max } \overline{z_{k}}\left(F_{k}^{+}+F_{k}^{-}\right)$

$$
\begin{gathered}
-\sum_{k}\left(1-\overline{z_{k}}\right) M_{k}\left(\mu_{k}^{+}+\mu_{k}^{-}\right)-\sum_{g} P_{g}^{\max } \alpha_{g} \\
\text { s.t.: } \sum_{k(n, .)}\left(\mu_{k}^{+}-\mu_{k}^{-}\right)-\sum_{k(., n)}\left(\mu_{k}^{+}-\mu_{k}^{-}\right)=0, \forall n
\end{gathered}
$$$$
B_{k}\left(L M P_{n}-L M P_{m}-F_{k}^{+}+F_{k}^{-}\right)
$$$$
-\mu_{k}^{+}+\mu_{k}^{-}=0, \forall k
$$

$L M P_{n}-\alpha_{g} \leq c_{g}, \forall g$

$F_{k}^{+}, F_{k}^{-}, \mu_{k}^{+}, \mu_{k}^{-}, \alpha_{g} \geq 0 ; L M P_{n}$ free

\section{Step 1: Initialize}

- Set $z_{k}$ to current topology configuration (must be feasible) or $z_{k}=1 \forall k$

- $\quad$ Upper Bound (UB) = infinity

- Lower Bound (LB) = -infinity

Step 2: Solve sub-problem for current fixed $z_{k}$ solution

- Unbounded sub-problem: add unbounded ray Benders' cut (23) to master problem and go to step 4

- Bounded sub-problem: obtain optimal solution and go to step 3

Step 3: Revenue adequacy check

- If revenue adequate, i.e., if (16a) holds, apply extreme point Benders' cut (22), update UB: $\mathrm{UB}=\min ($ current $\mathrm{UB}$, sub-problem optimal solution), and go to step 4

- If revenue inadequate, define sets in (25) based on current fixed $z_{k}$, apply cut (21) to the master problem, and go to step 4

Step 4: Solve master problem

- $\mathrm{LB}=$ new master problem optimal solution

- If UB-LB > epsilon, get new $z_{k}$ solution and go to step 2

- If UB-LB < epsilon, stop

\section{Test case and results}

Information on the test case used for this study can be found in [15]. We first assume that line 119 is out of service, which is explain at the end of this section. With line 119 out of service, the optimal cost is $\$ 2,114 / \mathrm{h}$ as compared to the optimal DCOPF solution with all lines in service, which has a cost of $\$ 2,054 / \mathrm{h}$. An FTR allocation is chosen that is revenue adequate for the static topology, i.e. all lines closed, and revenue inadequate by $12 \%$ with line 119 out of service.

For these studies, we restrict the number of lines that can be open to three. The optimal transmission switching problem takes roughly 7.5 minutes. The optimal solution is now $\$ 1817 / \mathrm{h}$. The revenue adequate optimal transmission switching problem finds the optimal revenue adequate solution in 9 minutes and after 176 iterations. The solution happens to be the same as the one found when revenue adequacy is not enforced; however, there is no guarantee that this generally will be true. The revenue adequate optimal transmission switching problem did not solve as fast as the original optimal transmission switching problem; however, a feasible revenue adequate solution with an objective at $\$ 1893 / \mathrm{h}$ was found in only 12 seconds. Testing would be needed on large-scale systems to further confirm this concept. 
We assumed line 119 to be out of service in order to explain a secondary point: just as altering the topology can cause revenue inadequacy, additional reconfigurations may regain revenue adequacy. In this case, the outage of line 119 can be thought of as a line failure that caused revenue inadequacy and then this concept is implemented to regain revenue adequacy.

\section{Policy implications}

Optimal transmission switching is a new concept that can be implemented by the central grid operator to achieve efficiency gains for the common good while maintaining reliability requirements. Unfortunately, implementation of such a concept can create winners and losers among market participants and it undermines a prevailing market assumption, that the grid is a static asset, required for the FTR market to operate as designed and desired. Thus, optimal transmission switching may cause a revenue shortfall in the FTR market but it can also create a revenue surplus. Since FTR settlements are cleared over multiple periods, there is the potential that the revenue shortfall over such a longer horizon is minimal. Nevertheless, examples have shown that revenue inadequacy can still be severe over long horizons, see [18], and, thus, we are still presented with the challenge of dealing with revenue inadequacy resulting from optimal transmission switching.

If optimal transmission switching causes revenue inadequacy in the FTR markets, ISOs may uniformly de-rate the FTR settlements. Such an approach to handle a revenue shortfall will obviously raise objections as it is not easy to convince FTR holders that their income should be decreased for the purpose of achieving efficiency gains for the common good. Another approach would be to implement side payments. With optimal transmission switching, the market surplus is guaranteed not to decrease so there is always the opportunity to implement side payments to make everyone at least as well off as they would be otherwise. However, there is then the following controversial question as to who should cover such a revenue shortfall. Uplift payments are often covered by consumers; however, past research on optimal transmission switching has demonstrated that, with a nodal pricing system, there is no guarantee that the consumers benefit, i.e., the total load payment reduces, as a result of optimal transmission switching, [15] and [16].

The ISO could instead require the beneficiaries to cover the revenue shortfall. However, identifying beneficiaries has been one of the major obstacles holding back transmission investments and, thus, such an obstacle is unlikely to be any easier to resolve in regards to the optimal transmission switching arena. Furthermore, there is yet another question as to what should be defined as the fair benchmark used to determine such side payments. Advances in technology will continue to change the market results and create winners and losers. Does it make sense to move forward with technology but yet require settlements based on a benchmark defined by an obsolete market design or to hold back an advance in technology since it may undermine an obsolete market design? As technology advances, so should the market design and market mechanisms, if necessary, but this does not prevent objections from market participants. The ISO itself may be conflicted against optimal transmission switching. Revenue inadequacy and the need to make uplift payments are often misjudged as a market failure. This situation is a manifestation of the complexity associated with the electric energy market structure and the difficulties in creating market mechanisms that can deal with such complexities.

These are all challenging policy issues. The revenue adequacy constrained optimal transmission switching concept provides an intermediate solution to such challenging problems. This concept adds to the optimal transmission switching problem the requirement to maintain revenue adequacy. Adding such a restriction may exclude, however, the true optimal network topology that maximizes the market surplus from being feasible, which amounts to "leaving money on the table" since, as stated earlier, the ISO could instead take the approach to maximize the market surplus but implement side payments if there is revenue inadequacy. However, it is easy to describe what should happen; it may not be as easy to actually formulate and implement such an agreement. As a result, this concept allows the operator to ensure revenue adequacy or, at least, limit the severity of revenue inadequacy, as shown by the use of $\delta$ in (16a). Furthermore, it is possible that the optimal network topology is revenue inadequate while there can be other topology solutions that are trivially more expensive but these topology solutions can have substantially different results regarding revenue adequacy, [18].

The ideal solution to this issue would be to redesign the FTR markets in order to resolve this issue. At the very least, this concept could be implemented by the ISO until the ISO auctions off the FTR positions at the next auction cycle, which can be once every six months. Once those participating in the FTR auctions understand the ability for optimal transmission switching to affect the value and the hedging ability of the FTRs, they will be able to take this into consideration when bidding on FTR positions. 


\section{Future research}

Future research should first examine a practical network with the assigned FTR positions and analyze whether revenue inadequacy would occur with optimal transmission switching. If revenue inadequacy is a potential problem, future research should examine whether the flexibility of the network can be accounted for in the FTR auction. Other future research topics include an investigation of a possible side payment mechanism and, ideally, a new financial derivative that would not be affected by optimal transmission switching but would still allow hedging of congestion risk.

Since the DCOPF is only a crude approximation of the ACOPF, future research is needed to examine optimal transmission switching with the ACOPF, its affect on reactive power, voltage stability, losses, etc. Optimal transmission switching provides another layer of control; thus, it provides a superset of feasible dispatch solutions. This is true no matter the structure of the OPF. With these facts along with previous research demonstrating the value of transmission control for AC systems as well as the existence of SPSs that implement pre-contingency and post-contingency switching actions, we are optimistic that the general conclusions will not change: that this added flexibility will benefit system operations.

There is, however, a practical challenge regarding the implementation of this concept. The ACOPF is a difficult, non-convex optimization problem. Combining a difficult integer program with the ACOPF is not practical today for large-scale problems. This is confirmed by the fact that unit commitment as well as transmission expansion planning problems are not combined with an ACOPF but with a DCOPF. A similar approach should be explored for the implementation of optimal transmission switching. Additional future research topics include: the need for circuit breaker control, monitoring, and maintenance, cost of switching, and whether frequent changing of relay settings will be necessary.

\section{Conclusion}

Recent research has demonstrated that we have yet to fully harness the flexibility that exists in the transmission network. Short term network topology reconfiguration can be beneficial for a variety of reasons, including voltage stability, loss reduction, reliability improvements, etc. One new concept in particular, optimal transmission switching, is capable of providing substantial economic savings by co- optimizing the network topology with generation while maintaining reliability requirements.

FTRs are important in electricity markets since they allow for hedging of congestion risk. Revenue inadequacy occurs when the ISO has a revenue shortfall and cannot cover its true obligation to the FTR holders. As a result, ISOs typically uniformly de-rate the FTR settlements, thereby decreasing the value of the FTR positions and breaking hedges that market participants hold in order to mitigate risk. One setback of the optimal transmission switching concept is that it may cause revenue inadequacy in the FTR market.

The implementation of new technologies under the umbrella of the smart grid may make current market mechanisms and protocols obsolete. Optimal transmission switching undermines a key assumption, that the transmission grid is a static asset, which is necessary for the FTR market to operate. The ideal solution to this issue would be to redesign the FTR market in some way to reflect the fact that the topology is, indeed, flexible and controllable by the grid operator while still being able to guarantee revenue adequacy. Another solution would be to implement a side payment scheme, when necessary, that would be agreed upon by market participants. At this time, however, such a redesign of the FTR market does not exist and the possibility to get market participants to approve a side-payment mechanism that covers such a revenue shortfall is unlikely.

Optimal transmission switching may or may not cause a revenue inadequacy problem. If it causes revenue inadequacy, then market participants may object to its implementation. Furthermore, since revenue inadequacy is often misjudged as a market failure, the ISOs themselves may be hesitant to implement it. This paper presents the concept of revenue adequacy constrained optimal transmission switching. While this concept should not be seen as a permanent solution to this issue, it is an affective intermediate solution that can be implemented, if desired by the ISO, until a more permanent remedy to this potential problem is created.

\section{References}

[1] O. Alsac, J. M. Bright, S. Brignone, M. Prais, C. Silva, B. Scott, and N. Vempati, "The rights to fight price volatility," IEEE Power and Energy Magazine, vol. 2, no. 4, pp. 47-57, 2004.

[2] R. Bacher and H. Glavitsch, "Network topology optimization with security constraints," IEEE Trans. Power Syst., vol. 1, no. 4, pp. 103-111, Nov. 1986.

[3] R. Bacher and H. Glavitsch, "Loss reduction by network switching," IEEE Trans. Power Syst., vol. 3, no. 2, pp. 447454, May 1988.

[4] A. G. Bakirtzis and A. P. (Sakis) Meliopoulos, "Incorporation of switching operations in power system 
corrective control computations," IEEE Trans. Power Syst., vol. 2, no. 3, pp. 669-675, Aug. 1987.

[5] S. Chen and H. Glavisch, "Stabilizing switching," IEEE Trans. Power Syst., vol. 8, no. 4, pp. 1511-1517, Nov. 1993.

[6] L. Chen, Y. Tada, H. Okamoto, R. Tanabe, and H. Mitsuma, "Optimal reconfiguration of transmission systems with transient stability constraints," in Proc. 1998 INTL. Conf. on Power Syst. Tech., vol. 2, pp. 1346-1350, 1998.

[7] G. Codato and M. Fischetti, "Combinatorial Benders' cuts for mixed-integer linear programming," Operations Research, vol. 54, no. 4, pp. 756-766, Aug. 2006.

[8] E. B. Fisher, R. P. O'Neill, and M. C. Ferris, "Optimal transmission switching," IEEE Trans Power Syst., vol. 23, no. 3, pp. 1346-1355, Aug. 2008.

[9] M. Fischetti and A. Lodi, "Local branching," Mathematical Programming, vol. 98, no. 1-3, pp. 23-47, Sept. 2003.

[10] S. Fliscounakis, F. Zaoui, G. Simeant, and R. Gonzalez, "Topology influence on loss reduction as a mixed integer linear programming problem," in IEEE Power Tech 2007, pp. 1987-1990, July 2007.

[11] H. Glavitsch, "State of the art review: switching as means of control in the power system," INTL. JNL. Elect. Power Energy Syst., vol. 7, no. 2, pp. 92-100, Apr. 1985.

[12] B. G. Gorenstin, L. A. Terry, M. V. F. Pereira, and L. M. V. G. Pinto, "Integrated network topology optimization and generation rescheduling for power system security applications," in IASTED INTL. SYMP.: High Tech. in the Power Industry, pp. 110-114, Bozeman, MT, Aug. 1986.

[13] G. Granelli, M. Montagna, F. Zanellini, P. Bresesti, R. Vailati, and M. Innorta, "Optimal network reconfiguration for congestion management by deterministic and genetic algorithms," Electr. Power Syst. Res., vol. 76, no. 6-7, pp. 549-556, Apr. 2006.

[14] K. W. Hedman, M. C. Ferris, R. P. O’Neill, E. B. Fisher, and S. S. Oren, "Co-optimization of generation unit commitment and transmission switching with $\mathrm{N}-1$ reliability," IEEE Trans. Power Syst., vol. 25, no. 2, pp. 1052-1063, May 2010.

[15] K. W. Hedman, R. P. O’Neill, E. B. Fisher, and S. S. Oren, "Optimal transmission switching - sensitivity analysis and extensions," IEEE Trans. Power Syst., vol. 23, no. 3, pp. 1469-1479, Aug. 2008.

[16] K. W. Hedman, R. P. O’Neill, E. B. Fisher, and S. S. Oren, "Optimal transmission switching with contingency analysis," IEEE Trans. Power Syst., vol. 24, no. 3, Aug. 2009.

[17] K. W. Hedman, R. P. O’Neill, E. B. Fisher, and S. S. Oren, "Smart flexible just-in-time transmission and flowgate bidding," IEEE Trans. Power Syst., accepted for publication, Mar. 2010.

[18] K. W. Hedman, S. S. Oren, and R. P. O'Neill, “Optimal transmission switching: economic efficiency and market implications," Journal of Regulatory Economics, submitted for publication, Apr. 2010.

[19] W. W. Hogan, "Contract Networks for Electric Power Transmission," Journal of Regulatory Economics, vol. 4, pp. 211-242, 1992.

[20] ISO-NE, ISO New England Operating Procedure no. 19: Transmission Operations, pp. 7-8, Apr. 13, 2007.
[21] A. A. Mazi, B. F. Wollenberg, and M. H. Hesse, "Corrective control of power system flows by line and busbar switching," IEEE Trans. Power Syst., vol. 1, no.3, pp. 258-264, Aug.1986.

[22] S. Nauman, VP, Exelon, Chicago, IL, private communication, July 2008.

[23] G. L. Nemhauser and L. A. Wolsey, Integer and Combinatorial Optimization. New York: Wiley-Interscience, 1999.

[24] Northeast Power Coordinating Council, Guidelines for inter-area voltage control. New York, NY: NPCC Operating Procedure Coordinating Committee and NPCC System Design Coordinating Committee, 1997.

[25] R. P. O'Neill, R. Baldick, U. Helman, M. H. Rothkopf, and W. Stewart, "Dispatchable transmission in RTO markets," IEEE Trans. Power Syst., vol. 20, no. 1, pp. 171179, Feb. 2005.

[26] R. P. O’Neill, K. W, Hedman, E. A. Krall, A. Papavasiliou, and S. S. Oren, "Economic analysis of the N-1 reliable unit commitment and transmission switching problem using duality concepts," Energy Systems Journal, vol. 1, no. 2, pp. 165-195, 2010.

[27] R. P. O’Neill, U. Helman, B. F. Hobbs, W. R. Stewart, Jr., and M. H. Rothkopf, "A joint energy and transmission rights auction for RTO markets," in Proc. IEE $5^{\text {th }}$ INTL Conf. on Power System Management and Control, London, April 2002.

[28] A. Ott, VP, PJM, Norristown, PA, private communication, July 2008.

[29] PJM, Manual 3: Transmission Operations, Revision: 35, October 5, 2009. Section 5: Index and Operating Procedures for PJM RTO Operation. [Online]. Available: http://www.pjm.com/markets-and-

operations/compliance/nerc-

standards/ /media/documents/manuals/m03.ashx

[30] W. Rei, M. Gendreau, J. F. Cordeau, and P. Soriano, "Accelerating Benders' decomposition by local branching," INFORMS Journal of Computing, vol. 21, no. 2, pp. 333-345, Spring 2009.

[31] G. Schnyder and H. Glavitsch, "Integrated security control using an optimal power flow and switching concepts," IEEE Trans. Power Syst., vol. 3, no. 2, pp. 782790, May 1988.

[32] G. Schnyder and H. Glavitsch, "Security enhancement using an optimal switching power flow," IEEE Trans. Power Syst., vol. 5, no. 2, pp. 674-681, May 1990.

[33] W. Shao and V. Vittal, "Corrective switching algorithm for relieving overloads and voltage violations," IEEE Trans. Power Syst., vol. 20, no. 4, pp. 1877-1885, Nov. 2005.

[34] W. Shao and V. Vittal, "BIP-based OPF for line and bus-bar switching to relieve overloads and voltage violations," in Proc. 2006 IEEE Power System Conf. and Expo., Atlanta, GA, Oct. 2006. 